\title{
Frecuencia de Streptococcus pneumoniae aislados de enfermedad invasiva en Paraguay, serotipos y perfil de sensibilidad (2010-2018)
}

\author{
*María Eugenia León ${ }^{1}$, Aníbal Kawabata ${ }^{1}$, Minako Nagai ${ }^{1}$, Liliana Rojas ${ }^{1}$, Noemí Zárate ${ }^{2}$, Juan Irala ${ }^{3}$, \\ Gloria Gómez ${ }^{4}$, Juana Ortellado ${ }^{5}$, Myrian Leguizamón ${ }^{6}$, Rossana Franco ${ }^{7}$ Nancy Segovia ${ }^{8}$, Gustavo \\ Chamorro $^{1}$ \\ ${ }^{1}$ Ministerio de Salud Pública y Bienestar Social, Laboratorio Central de Salud Pública. Paraguay \\ ${ }^{2}$ Hospital General Pediátrico Niños de Acosta Ñu. San Lorenzo, Paraguay \\ ${ }^{3}$ Instituto de Medicina Tropical. Paraguay \\ ${ }^{4}$ Hospital Nacional de Itaugua. Paraguay \\ ${ }^{5}$ Universidad Nacional de Asunción, Hospital de Clínicas. San Lorenzo, Paraguay \\ ${ }^{6}$ Instituto de Previsión Social. Paraguay \\ ${ }^{7}$ Instituto Nacional de Enfermedades Respiratorias y del Ambiente. Paraguay \\ ${ }^{8}$ Hospital Regional Ciudad del Este. Paraguay
}

\begin{abstract}
Cómo referenciar este artículo/
\end{abstract}
How to reference this article:
León ME, Kawabata A, Nagai M, Rojas L, Zárate N, Irala J, et al. Frecuencia de Streptococcus pneumoniae aislados de enfermedad invasiva en Paraguay, serotipos y perfil de sensibilidad (20102018). Mem. Inst. Investig. Cienc. Salud. 2020; 18(1): 38-46

\section{R E S U M E N}

Streptococcus pneumoniae sigue siendo una de las causas más importantes de morbilidad y mortalidad en niños y adultos alrededor del mundo. El objetivo del estudio fue describir la frecuencia de aislamiento de $S$. pneumoniae en enfermedad invasiva, distribución de serotipos y sensibilidad antimicrobiana en Paraguay (2010-2018). Se estudiaron 793 cepas de $S$. pneumoniae aisladas de pacientes de todas las edades con enfermedad invasiva en Paraguay, provenientes de los diferentes centros centinelas y colaboradores en el marco de la vigilancia de meningitis y neumonías, durante el periodo 2010-2018. La frecuencia general según diagnóstico resultó $74.9 \%$ de neumonías $(n=594), 18.4 \%$ de meningitis $(n=146)$ y $6.7 \%$ de sepsis $(n=53)$. El serotipo 14 fue más frecuente con 174 aislamientos $(22.0 \%)$, seguido del serotipo 19A con 84 aislamientos $(10.6 \%)$, el serotipo 3 con 66 aislamientos $(8.3 \%)$ y el $6 \mathrm{~A}$ con 37 aislamientos $(4.7 \%)$. En meningitis se registró una frecuencia general de resistencia a penicilina del $32,2 \%$ y de ceftriaxona del $1,4 \%$. En los casos de no meningitis la resistencia a penicilina fue del $0,8 \%$ y ceftriaxona del $0,3 \%$. Los resultados de serotipos y sensibilidad antimicrobiana proporcionarán información necesaria para la implementación de estrategias de prevención y tratamiento de la enfermedad neumocócica en nuestro país, por lo que es necesaria una vigilancia continua para evaluar la carga de enfermedad, los serotipos circulantes y el aumento de la resistencia a los antibióticos.

Palabras clave: Streptococcus pneumoniae, enfermedad invasiva, serotipos, perfil de sensibilidad.

\section{Frequency of Streptococcus pneumoniae isolated from invasive disease in Paraguay, serotypes and sensitivity profile (2010-2018)}

\section{A B S T R A C T}

Streptococcus pneumoniae remains one of the most important causes of morbidity and mortality in children and adults worldwide. The objective of the study was to describe the frequency of isolation of $S$. pneumoniae in invasive disease, serotype distribution and antimicrobial susceptibility in Paraguay (2010-2018). We studied 793 strains of $S$. 
pneumoniae isolated from patients of all ages with invasive disease in Paraguay, from different sentinel centers and collaborators in the framework of meningitis and pneumonia surveillance during the period 2010-2018. The general frequency according to diagnosis was $74.9 \%$ of pneumonia $(n=594), 18.4 \%$ of meningitis $(n=146)$ and $6.7 \%$ of sepsis $(n=$ $53)$. Serotype 14 was more frequent with 174 isolates $(22.0 \%)$, followed by serotype $19 \mathrm{~A}$ with 84 isolates $(10.6 \%)$, serotype 3 with 66 isolates $(8.3 \%)$ and $6 \mathrm{~A}$ with 37 isolates $(4.70 \%)$. In meningitis, there was a general frequency of penicillin resistance of $32.2 \%$ and ceftriaxone of $1.4 \%$. In cases of non-meningitis, penicillin resistance was $0.8 \%$ and ceftriaxone $0.3 \%$. The results of serotypes and antimicrobial sensitivity will provide necessary information for the implementation of prevention strategies and treatment of pneumococcal disease in our country, therefore it is necessary to continue monitoring in order to assess the burden of the disease, circulating serotypes and increased antibiotic resistance.

Keywords: Streptococcus pneumoniae, invasive disease, serotypes, sensitivity profile.

\section{INTRODUCCIÓN}

Streptococcus pneumoniae sigue siendo una de las causas más importantes de morbilidad y mortalidad en niños y adultos alrededor del mundo ${ }^{(1)}$, anualmente mueren 1.1 millones de niños menores de cinco años a causa de infecciones neumocócicas y casi todas estas muertes ocurren en niños de países de ingresos bajos y medios bajos ${ }^{(2)}$. Es el principal agente causal de enfermedades invasivas graves como meningitis, neumonías y sepsis.

Su principal factor de virulencia es la cápsula polisacárida que proporciona protección frente a la fagocitosis e inhibe la actividad del complemento y permite la clasificación en diferentes serotipos. Se han identificado más de 93 serotipos ${ }^{(3)}$, pero solo unos pocos causan la mayoría de las enfermedades invasivas. Los serotipos más prevalentes han ido cambiando desde la introducción de las vacunas ya que son serotipo especificas ${ }^{(4,5)}$.

Existen vacunas conjugadas para disminuir la carga de enfermedad y combatir el aumento de la resistencia antimicrobianos. La disponibilidad de las mismas ha reducido la enfermedad neumocócica y las muertes en los países donde se incorporaron al calendario de vacunación nacional ${ }^{(6)}$. No obstante, existen preocupaciones sobre sus efectos a largo plazo, ya que contienen un número limitado de serotipos y el papel potencial de los serotipos que no son vacunas. En el 2012, la vacuna neumocócica conjugada 10-valente, que contiene los serotipos 1,4, 5, 6B, 7F, 9V, 14, 18C, 19F y $23 \mathrm{~F}$ de $S$. pneumoniae, fue incorporada al esquema regular de vacunación nacional de Paraguay.

La penicilina ha sido el tratamiento de elección durante muchos años pero el aumento de la incidencia de enfermedad neumocócica causada por cepas resistentes a los antibióticos hace que estas infecciones comunes sean un problema aún más grave y se dificulte el tratamiento de la enfermedad ${ }^{(7,8)}$.

En 1998 se inicia la vigilancia de S. pneumoniae (serotipos capsulares y susceptibilidad antimicrobiana) en Paraguay, a través del proyecto del Sistema Regional de Vacunas (SIREVA) de la Organización Panamericana de la Salud (OPS), en niños menores de 6 años y en población general. En el 2003 se integra al sistema de vigilancia de enfermedades transmisibles como VIMENE (vigilancia nacional de meningitis y neumonías). Según la Dirección General de Vigilancia de la Salud, en Paraguay S. pneumoniae causa el 37,72\% del total de meningitis bacterianas ${ }^{(9)}$. El serotipo 14 resultó el más frecuente en niños menores de 6 años entre los años 2000 y 2008 y se reportó una resistencia a penicilina del $12,1 \%{ }^{(10)}$. En adultos se halló una prevalencia del serotipo 3 y resistencia a la penicilina del $38,5 \%{ }^{(11)}$. En América Latina y el Caribe El serotipo 14 de $S$. pneumoniae fue el más frecuentemente aislado $(21,1 \%)$, especialmente en niños menores de 6 años $(29,1 \%)$ y se encontró una resistencia a la penicilina del $15,4 \%{ }^{(12)}$.

El objetivo del estudio fue describir la frecuencia de aislamiento de $S$. pneumoniae en enfermedad neumocócica invasiva, distribución de serotipos y sensibilidad antimicrobiana en Paraguay (2010-2018).

\section{MATERIALES Y MÉTODOS}

Estudio descriptivo, observacional, retrospectivo, de corte transversal. Se estudiaron 793 cepas de $S$. pneumoniae aisladas de pacientes de todas las edades con enfermedad invasiva en Paraguay, provenientes de los diferentes centros centinelas y colaboradores en el marco 
de la vigilancia de meningitis y neumonías, durante el periodo 2010-2018. El análisis se realizó utilizando Epi-info versión 7.2. Este estudio se limitó a descripción de los datos, sin incluir ningún análisis de inferencia estadística.

\section{Identificación de los aislamientos}

Los aislamientos de $S$. pneumoniae se identificaron mediante procedimientos de laboratorio estandarizados, como la morfología de la colonia en agar sangre, la presencia de alfa-hemólisis, la prueba de sensibilidad a la optoquina (Oxoid, Basingstoke, Reino Unido) y solubilidad en bilis. La serotipificación capsular se realizó mediante la reacción de Quellung (antisueros del Statens Serum Institut, Copenhagen, Denmark) según recomendaciones de la red SIREVA-OPS/OMS ${ }^{(13)}$.

\section{Pruebas de susceptibilidad antimicrobiana}

Los aislamientos bacterianos se analizaron mediante el método de difusión en disco según las recomendaciones del Instituto de Estándares Clínicos y de Laboratorio ${ }^{(14)}$. Los agentes antimicrobianos analizados fueron: cloranfenicol (30 $\mu \mathrm{g})$, eritromicina (15 $\mu \mathrm{g})$, sulfametoxazol-trimetoprim $(23.75 \mu \mathrm{g} / 1.25) \mu \mathrm{g})$, tetraciclina $(30 \mu \mathrm{g})$ y vancomicina (30 $\mu \mathrm{g})$. Todos los aislamientos se sometieron a la determinación de las concentraciones inhibitorias mínimas (MIC) de penicilina y ceftriaxona mediante el uso de las tiras E-test (BioMérieux, Francia).

\section{Consideraciones éticas}

El estudio se llevó a cabo de acuerdo con los lineamientos éticos señalados en la Declaración de Helsinki. Este estudio fue aprobado por el Comité de Ética en Investigación del Laboratorio Central de Salud Pública (CEI). No se obtuvo declaración de consentimiento informado debido a que el estudio es observacional, con riesgo mínimo o nulo para los sujetos y abarcó un periodo muy largo durante el cual fue imposible recoger los consentimientos informados de todos los sujetos del estudio. La confidencialidad de los datos que consignan a la persona se respetó en todo momento y únicamente los investigadores pudieron acceder a los datos referidos.

\section{RESULTADOS}

Se estudiaron 793 cepas de S. pneumoniae aisladas de 594 muestras de líquido pleural, 146 muestras de líquido cefalorraquídeo y 53 muestras de sangre, las mismas fueron obtenidas de 460 pacientes del sexo masculino y 333 del sexo femenino. Se observó una frecuencia de aislamiento de $S$. pneumoniae del $48.42 \%$ en $<5$ años $(n=384)$, seguido del $12.10 \%$ en $>60$ años $(n=96)$ (Tabla 1$)$.

Tabla 1: Frecuencia de aislamiento de S. pneumoniae por año, según sexo y edad (20102018) $(n=793)$

\begin{tabular}{|c|c|c|c|c|c|c|c|c|c|c|c|c|c|c|c|c|c|c|c|c|}
\hline \multirow[b]{3}{*}{ Año } & & & \multicolumn{4}{|c|}{ Sexo } & \multicolumn{14}{|c|}{ Edad (en años) } \\
\hline & \multicolumn{2}{|c|}{$\begin{array}{l}\text { Número de } \\
\text { cassos }\end{array}$} & \multicolumn{2}{|c|}{ Femenino } & \multicolumn{2}{|c|}{ Masculino } & \multicolumn{2}{|c|}{$<5$} & \multicolumn{2}{|c|}{$5-14$} & \multicolumn{2}{|c|}{$15-29$} & \multicolumn{2}{|c|}{$30-49$} & \multicolumn{2}{|c|}{$50-59$} & \multicolumn{2}{|c|}{$>60$} & \multicolumn{2}{|c|}{ Sin datos } \\
\hline & $n$ & $\%$ & $n$ & $\%$ & $n$ & $\%$ & $n$ & $\%$ & $n$ & $\%$ & $n$ & $\%$ & $n$ & $\%$ & $n$ & $\%$ & $n$ & $\%$ & $\mathrm{n}$ & $\%$ \\
\hline 2010 & 121 & 15,26 & 52 & 42,98 & 69 & 57,02 & 71 & 58,68 & 11 & 9,09 & 7 & 5,78 & 10 & 8,26 & 1 & 0,83 & 4 & 3,30 & 17 & 14,05 \\
\hline 2011 & 103 & 12,99 & 53 & 51,46 & 50 & 48,54 & 57 & 55,34 & 16 & 15,53 & 3 & 2,91 & 9 & 8,74 & 6 & 5,82 & 12 & 11,65 & 0 & 0,00 \\
\hline 2012 & 96 & 12,11 & 43 & 44,79 & 53 & 55,21 & 41 & 42,71 & 11 & 11,46 & 8 & 8,33 & 11 & 11,46 & 5 & 5,21 & 12 & 12,50 & 8 & 8,33 \\
\hline 2013 & 78 & 9,84 & 29 & 37,18 & 49 & 62,82 & 36 & 46,15 & 8 & 10,26 & 5 & 6,41 & 6 & 7,69 & 8 & 10,26 & 10 & 12,82 & 5 & 6,41 \\
\hline 2014 & 58 & 7,31 & 26 & 44,83 & 32 & 55,17 & 23 & 39,65 & 8 & 13,79 & 7 & 12,07 & 3 & 5,17 & 0 & 0,00 & 7 & 12,07 & 10 & 17,24 \\
\hline 2015 & 64 & 8,07 & 20 & 31,25 & 44 & 68,75 & 29 & 45,31 & 10 & 15,62 & 2 & 3,12 & 5 & 7,81 & 4 & 6,25 & 8 & 12,50 & 6 & 9,37 \\
\hline 2016 & 86 & 10,84 & 37 & 43,02 & 49 & 56,98 & 36 & 41,86 & 9 & 10,46 & 5 & 5,81 & 11 & 12,79 & 5 & 5,81 & 10 & 11,63 & 10 & 11,63 \\
\hline 2017 & 91 & 11,47 & 36 & 39,56 & 55 & 60,44 & 47 & 51,65 & 8 & 8,79 & 5 & 5,49 & 8 & 8,79 & 7 & 7,69 & 15 & 16,48 & 1 & 1,10 \\
\hline 2018 & 96 & 12,11 & 37 & 38,54 & 59 & 61,46 & 44 & 45,83 & 7 & 7,29 & 6 & 6,25 & 14 & 14,58 & 7 & 7,29 & 18 & 18,75 & 0 & 0,00 \\
\hline Total & 793 & 100,00 & 333 & 42,00 & 460 & 58,00 & 384 & 48,42 & 88 & 11,10 & 48 & 6,00 & 77 & 9,70 & 43 & 5,42 & 96 & 12,10 & 57 & 7,18 \\
\hline
\end{tabular}

La frecuencia general según diagnóstico resultó $74.9 \%$ de neumonías $(n=594), 18.4 \%$ de meningitis $(n=146)$ y $6.7 \%$ de sepsis $(n=53)$. En el año 2010 se observó mayor número de casos de meningitis $(n=27)$, en el año 2011 mayor número de casos de neumonías $(n=86)$ y en los años 2010 y 2018 se observó mayor número de casos de sepsis $(n=13)$ respectivamente (Figura 1 ). 


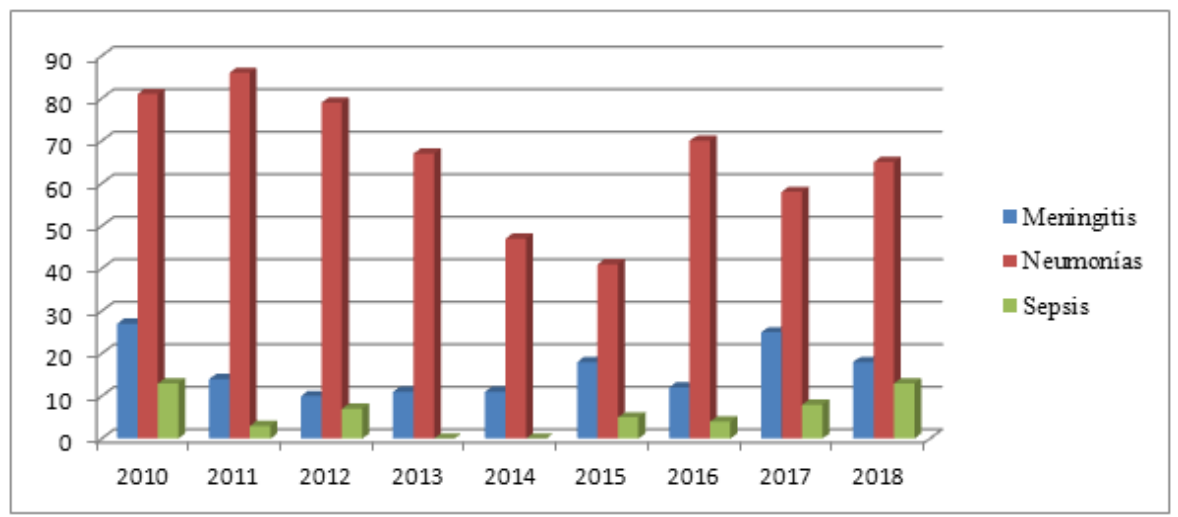

Figura 1: Frecuencia de aislamientos de S. pneumoniae según diagnóstico por año (20102018) $(n=793)$.

Se encontraron 47 serotipos diferentes. El serotipo 14 fue más frecuente con 174 aislamientos (22.0\%), seguido del serotipo $19 \mathrm{~A}$ con 84 aislamientos $(10.6 \%)$, el serotipo 3 con 66 aislamientos (8.3\%) y el $6 \mathrm{~A}$ con 37 aislamientos (4.7\%). Además se aislaron 123 cepas que corresponden a los serotipos no vacunales $(6 C, 6 D, 7 A, 7 C, 9 A, 9 L, 12 A, 13$, 15A, 15F, 16F, 18A, 18B, 18F, 19B, 19C, 22A, 23A, 23B, 24F, 34, 35B Y 35F), entre estos los serotipos más frecuentes son el $23 B(n=21), 24 F(n=9)$ y $23 A(n=6)$. Tabla 2.

La distribución de serotipos según grupo de edad mostró que en niños menores de 5 años fueron aislados con mayor frecuencia el serotipo $14(35,0 \%)$, seguido del $19 \mathrm{~A}(16,0 \%)$, del serotipo $3(15,0 \%), 6 \mathrm{~A}(9,0 \%), 6 \mathrm{~B}(7,0 \%)$ y $12 \mathrm{~F}(4,0 \%)$. En adultos mayores de 60 años se encontró una mayor frecuencia del serotipo $19 \mathrm{~A}(17,0 \%)$, seguido del $14(13,0 \%), 23 \mathrm{~B}$ $(12,0 \%)$, del $3(11,0 \%), 6 \mathrm{~A}(8,0 \%)$ y los serotipos $6 \mathrm{C}$, $7 \mathrm{~F}$ y $12 \mathrm{~F}(7,0 \%)$.

Tabla 2: Distribución de serotipos de S. pneumoniae aislados de enfermedad invasiva $(2010-2018)(n=793)$

\begin{tabular}{|c|c|c|c|c|c|c|c|c|c|c|}
\hline Serotipos & 2010 & 2011 & 2012 & 2013 & 2014 & 2015 & 2016 & 2017 & 2018 & Total \\
\hline 1 & 6 & 3 & 1 & 0 & 0 & 0 & 0 & 0 & 0 & 10 \\
\hline 4 & 5 & 1 & 0 & 0 & 0 & 0 & 0 & 0 & 0 & 6 \\
\hline 5 & 1 & 1 & 3 & 1 & 0 & 0 & 0 & 0 & 0 & 6 \\
\hline 6B & 13 & 6 & 4 & 4 & 5 & 2 & 2 & 0 & 0 & 36 \\
\hline $7 F$ & 7 & 2 & 2 & 3 & 0 & 3 & 2 & 3 & 0 & 22 \\
\hline 9V & 1 & 1 & 2 & 0 & 2 & 2 & 3 & 2 & 0 & 13 \\
\hline 14 & 44 & 42 & 33 & 15 & 7 & 9 & 13 & 5 & 6 & 174 \\
\hline $18 \mathrm{C}$ & 1 & 5 & 1 & 0 & 0 & 1 & 0 & 0 & 0 & 8 \\
\hline $19 \mathrm{~F}$ & 1 & 6 & 8 & 4 & 2 & 1 & 1 & 0 & 1 & 24 \\
\hline $23 \mathrm{~F}$ & 2 & 5 & 4 & 2 & 1 & 4 & 1 & 0 & 2 & 21 \\
\hline 3 & 0 & 1 & 1 & 0 & 13 & 5 & 11 & 19 & 16 & 66 \\
\hline 6A & 3 & 7 & 3 & 8 & 0 & 4 & 4 & 3 & 5 & 37 \\
\hline 19A & 7 & 3 & 3 & 3 & 2 & 8 & 15 & 18 & 25 & 84 \\
\hline 2 & 0 & 0 & 0 & 0 & 1 & 0 & 0 & 0 & 0 & 1 \\
\hline 8 & 1 & 2 & 0 & 3 & 2 & 0 & 2 & 0 & 1 & 11 \\
\hline $9 \mathrm{~N}$ & 1 & 0 & 0 & 0 & 0 & 0 & 3 & 0 & 0 & 4 \\
\hline $10 \mathrm{~A}$ & 0 & 0 & 0 & 0 & 0 & 1 & 1 & 0 & 0 & 2 \\
\hline 11A & 0 & 1 & 0 & 0 & 0 & 0 & 1 & 3 & 0 & 5 \\
\hline $12 \mathrm{~F}$ & 2 & 2 & 1 & 0 & 1 & 2 & 5 & 5 & 9 & 27 \\
\hline 15B & 0 & 1 & 2 & 0 & 4 & 0 & 2 & 1 & 1 & 11 \\
\hline $17 \mathrm{~F}$ & 1 & 0 & 1 & 1 & 0 & 0 & 1 & 1 & 1 & 6 \\
\hline 20 & 0 & 1 & 0 & 1 & 0 & 0 & 0 & 0 & 0 & 2 \\
\hline $22 F$ & 0 & 2 & 0 & 0 & 0 & 0 & 0 & 2 & 6 & 10 \\
\hline $33 F$ & 2 & 0 & 0 & 0 & 0 & 0 & 1 & 1 & 0 & 4 \\
\hline $\begin{array}{l}\text { NT } \\
\text { Otros }\end{array}$ & 7 & 4 & 17 & 22 & 10 & 8 & 1 & 0 & 0 & 69 \\
\hline $\begin{array}{c}\text { Serotipos no } \\
\text { vacunales }\end{array}$ & 10 & 7 & 10 & 9 & 7 & 14 & 15 & 28 & 23 & 123 \\
\hline $\begin{array}{c}\text { NV } \\
\text { Total }\end{array}$ & $\begin{array}{c}6 \\
121\end{array}$ & $\begin{array}{c}0 \\
103\end{array}$ & $\begin{array}{c}0 \\
96\end{array}$ & $\begin{array}{c}2 \\
78\end{array}$ & $\begin{array}{c}1 \\
58\end{array}$ & $\begin{array}{c}0 \\
64\end{array}$ & $\begin{array}{c}2 \\
86\end{array}$ & $\begin{array}{c}0 \\
91\end{array}$ & $\begin{array}{c}0 \\
96\end{array}$ & $\begin{array}{c}11 \\
793\end{array}$ \\
\hline
\end{tabular}

En meningitis se registró una frecuencia general de resistencia a penicilina del 32,2\%. Además se observó que el $1,4 \%$ de los aislamientos tuvieron sensibilidad intermedia a ceftriaxona y el $0,7 \%$ fueron resistentes (Tabla 3 ). Se observó un aumento de la resistencia a penicilina durante el periodo de estudio. Los serotipos asociados a la resistencia de pencilina fueron el $14,19 \mathrm{~A}, 23 \mathrm{~B}$ y el $6 \mathrm{~A}$. 
Tabla 3: Perfil de sensibilidad a la penicilina y ceftriaxona de $S$. pneumoniae aislados de meningitis por año $(n=146)$

\begin{tabular}{|c|c|c|c|c|c|c|}
\hline \multicolumn{6}{|c|}{ Frecuencia de sensibilidad a los antibióticos (\%) } \\
\hline & & \multicolumn{2}{|c|}{ Penicilina } & \multicolumn{3}{c|}{ Ceftriaxona } \\
\hline Año & $\begin{array}{c}\mathbf{N}^{\circ} \mathbf{d e} \\
\text { aislamientos }\end{array}$ & Sensible & Resistente & Sensible & Intermedio & Resistente \\
\hline 2010 & 27 & $19(70,4)$ & $8(29,6)$ & $26(96,3)$ & $0(0,0)$ & $1(3,7)$ \\
\hline 2011 & 14 & $11(78,6)$ & $3(21,4)$ & $13(92,8)$ & $1(7,2)$ & $0(0,0)$ \\
\hline 2012 & 10 & $7(70,0)$ & $3(30,0)$ & $9(90,0)$ & $1(10,0)$ & $0(0,0)$ \\
\hline 2013 & 11 & $9(81,8)$ & $2(18,2)$ & $11(100,0)$ & $0(0,0)$ & $0(0,0)$ \\
\hline 2014 & 11 & $8(72,7)$ & $3(27,3)$ & $11(100,0)$ & $0(0,0)$ & $0(0,0)$ \\
\hline 2015 & 18 & $12(66,7)$ & $6(33,3)$ & $18(100,0)$ & $0(0,0)$ & $0(0,0)$ \\
\hline 2016 & 12 & $8(66,7)$ & $4(33,3)$ & $12(100,0)$ & $0(0,0)$ & $0(0,0)$ \\
\hline 2017 & 25 & $15(60,0)$ & $10(40,0)$ & $25(100,0)$ & $0(0,0)$ & $0(0,0)$ \\
\hline 2018 & 18 & $10(55,5)$ & $8(44,5)$ & $18(100,0)$ & $0(0,0)$ & $0(0,0)$ \\
\hline Total & $\mathbf{1 4 6}$ & $\mathbf{9 9 ( 6 7 , 8 )}$ & $\mathbf{4 7 ( 3 2 , 2 )}$ & $\mathbf{1 4 3 ( 9 7 , 9 )}$ & $\mathbf{2 ( 1 , 4 )}$ & $\mathbf{1}(0,7)$ \\
\hline
\end{tabular}

a Según Concentración Inhibitoria Mínima (CIM): Sensible CIM $\leq 0,06 \mathrm{ug} / \mathrm{mL}$ Resistente $\geq 0,125 \mathrm{ug} / \mathrm{mL}$ (CLSI). b Según Concentración Inhibitoria Mínima (CIM): Sensible CIM $\leq 0,5 \mathrm{ug} / \mathrm{mL}$, Intermedio CIM $1 \mathrm{ug} / \mathrm{mL}$ y Resistente $\mathrm{CIM} \geq 2 \mathrm{ug} / \mathrm{mL}(\mathrm{CLSI})$.

En los aislamientos provenientes de neumonías y sepsis (no meningitis) se halló para penicilina una sensibilidad intermedia de $0,6 \%$ y resistencia de $0,8 \%$. Para ceftriaxona $1,4 \%$ de sensibilidad intermedia y $0,3 \%$ de resistencia (Tabla 4 ). Los serotipos asociados con mayor frecuencia a la resistencia de penicilina fueron el 14 y el 19A.

En meningitis y no meningitis observó una resistencia del $26,0 \%$ en eritromicina, $33,0 \%$ en trimetoprim-sulfametoxazol, $32,0 \%$ en tetraciclina y $2,0 \%$ en cloranfenicol.

Tabla 4: Perfil de sensibilidad a la penicilina y ceftriaxona de Streptococcus pneumoniae aislados de neumonías y sepsis por año $(n=636)$

\begin{tabular}{|c|c|c|c|c|c|c|c|}
\hline \multirow[b]{3}{*}{ Año } & \multicolumn{6}{|c|}{ Frecuencia de sensibilidad a los antibióticos (\%) } & \multirow[b]{3}{*}{ Resistente } \\
\hline & \multirow[b]{2}{*}{$\begin{array}{c}\mathrm{N}^{\circ} \mathrm{de} \\
\text { aislamientos }\end{array}$} & \multicolumn{3}{|c|}{ Penicilina } & \multicolumn{2}{|r|}{ Ceftriaxona } & \\
\hline & & Sensible & Intermedio & Resistente & Sensible & Intermedio & \\
\hline 2010 & 88 & $86(97,7)$ & $0(0,0)$ & $2(2,3)$ & $87(98,9)$ & $1(1,1)$ & $0(0,0)$ \\
\hline 2011 & 89 & $88(98,9)$ & $1(1,1)$ & $0(0,0)$ & $85(95,5)$ & $4(4,5)$ & $0(0,0)$ \\
\hline 2012 & 86 & $84(97,6)$ & $1(1,2)$ & $1(1,2)$ & $82(95,3)$ & $3(3,5)$ & $1(1,2)$ \\
\hline 2013 & 65 & $65(100,0)$ & $0(0,0)$ & $0(0,0)$ & $65(100,0)$ & $0(0,0)$ & $0(0,0)$ \\
\hline 2014 & 46 & $44(95,6)$ & $1(2,2)$ & $1(2,2)$ & $45(97,8)$ & $0(0,0)$ & $1(2,2)$ \\
\hline 2015 & 46 & $46(100,0)$ & $0(0,0)$ & $0(0,0)$ & $46(100,0)$ & $0(0,0)$ & $0(0,0)$ \\
\hline 2016 & 72 & $71(98,6)$ & $0(0,0)$ & $1(1,4)$ & $72(100,0)$ & $0(0,0)$ & $0(0,0)$ \\
\hline 2017 & 66 & $65(98,5)$ & $1(1,5)$ & $0(0,0)$ & $65(98,5)$ & $1(1,5)$ & $0(0,0)$ \\
\hline 2018 & 78 & $78(100,0)$ & $0(0,0)$ & $0(0,0)$ & $78(100,0)$ & $0(0,0)$ & $0(0,0)$ \\
\hline Total & 636 & $627(98,6)$ & $4(0,6)$ & $5(0,8)$ & $625(98,3)$ & $9(1,4)$ & $2(0,3)$ \\
\hline
\end{tabular}




\section{DISCUSIÓN}

EI S. pneumoniae representa un problema de salud pública especialmente en los países en desarrollo debido a la alta carga de enfermedad especialmente en el contexto de los programas de vacunación.

En este estudio se encontró una mayor frecuencia de aislamiento en niños menores de 5 años y adultos mayores de 60 años. Esta frecuencia fue similar a la hallada en diferentes países de la región ${ }^{(15)}$. Las neumonías y las meningitis fueron los diagnósticos más frecuentes durante este periodo de estudio, así como datos reportados en América Latina y el Caribe ${ }^{(16)}$.

La enfermedad neumocócica invasiva se asoció con mayor frecuencia al serotipo 14, similar a otros estudios realizados anteriormente ${ }^{(17,18)}$ y ha sido asociado particularmente con las enfermedades infecciosas en niños. Este serotipo está además asociado a la resistencia de los antimicrobianos ${ }^{(19)}$.

El serotipo 19A le sigue en frecuencia de aislamiento y los resultados indican que la frecuencia aumentó en los años posteriores a la introducción de la vacuna (2012). En Latinoamérica una revisión sistemática de Castañeda et al. en el periodo 1990-2010 mostró que el serotipo 19A ocupó el noveno lugar entre los 20 serotipos más prevalentes ${ }^{(20)}$. Otro estudio mostró que la proporción de aislamientos del serotipo 19A se duplicó en comparación con el número total de aislamientos ${ }^{(21)}$.

El serotipo 3 encontrado en esta vigilancia también registró un aumento posterior a la vacunación. Este cambio se ha registrado en estudios realizados en varios países ${ }^{(22,23)}$. Es importante destacar que este serotipo se ha asociado en gran medida con una alta mortalidad de adultos ${ }^{(24)}$. Existe evidencia sustancial de la limitada eficacia de las vacunas neumocócicas sobre el serotipo 3. Un trabajo de investigación sugiere que la cápsula del serotipo 3 inusualmente densa y gruesa puede eludir la protección del sistema inmunológico ${ }^{(25)}$.

Entre los serotipos no vacunales el $12 \mathrm{~F}$ se identificó con mayor frecuencia y se ha demostrado que el serotipo $12 \mathrm{~F}$ causa brotes en poblaciones humanas con factores de riesgo identificables ${ }^{(26)}$.

Un hallazgo importante de este estudio es la disminución anual del número de serotipos causantes de enfermedad invasiva incluidos en la PCV10. Una investigación realizada en México demostró que la proporción de serotipos vacunales disminuyó en todos los grupos de edad donde los serotipos de la PCV10 disminuyeron $2.6 \%$ en promedio por año ${ }^{(27)}$.

El aumento de las infecciones neumocócicas asociadas con cepas resistentes a los antibióticos ha impulsado mejores estrategias de prevención y control de la enfermedad. Los resultados de nuestro estudio arrojaron que los serotipos asociados con mayor frecuencia a la resistencia de penicilina fueron el 14 y el 19A. Según un estudio de aislamientos clínicos de $S$. pneumoniae en poblaciones globales, los serotipos 19A, 19F, $35 \mathrm{~B}, 6 \mathrm{~A}, 6 \mathrm{~B}, 23 \mathrm{~A}, 9 \mathrm{~V}, 15 \mathrm{~A}$ y 14 demostraron tasas más altas de la resistencia a la penicilina $(P<0.05)^{(28)}$.

La resistencia global hallada en este estudio de penicilina (en meningitis) fue del $32,2 \%$ y ceftriaxona del $0,7 \%$. Varios autores informaron tasas de resistencia a la penicilina de $S$. pneumoniae, aisladas de pacientes con meningitis, que oscilaron entre el $21,4 \%$ y el $27,1 \%$

(29). Un estudio basado en datos de vigilancia de laboratorio de 10 países latinoamericanos mostró que para meningitis el $30,5 \%$ de los aislamientos resultaron resistentes ${ }^{(30)}$. En muchos países de América Latina, la resistencia total a la ceftriaxona en los aislamientos de meningitis neumocócica es baja, reportándose el mayor porcentaje observado en México $(32.3 \%)^{(29)}$. Además, a lo largo del estudio se observa un aumento de la resistencia a penicilinas para los casos de meningitis, lo que refleja el aumento de la resistencia a la penicilina documentado en todo el mundo(31-34).

Las limitaciones de este estudio corresponden a la baja sensibilidad del cultivo microbiológico, si bien es el estándar de oro, tiene una recuperación de alrededor del $60 \%$ de los aislamientos. El tratamiento antibiótico previo a la toma de muestra y la demora entre la toma de muestra y el procesamiento afectan directamente la viabilidad de la bacteria, por lo que podría haber subregistros de casos en el país.

La vigilancia laboratorial y epidemiológica continua es necesaria para evaluar la carga de enfermedad, los serotipos circulantes y el aumento de la resistencia a los antibióticos en Paraguay, así como para las estrategias de inmunización con las vacunas actuales. 
Es importante fortalecer los programas de vigilancia epidemiológica con la implementación de técnicas moleculares que contribuirán a mejorar la sensibilidad del diagnóstico de meningitis y neumonías bacterianas, lo que ayudará a reconocer con más aproximación la carga real de la enfermedad en nuestro país, lo que redundará en mejorar las estrategias de los programas de promoción y prevención.

\section{AGRADECIMIENTOS}

La realización de este trabajo fue apoyada por subvenciones del Fondo para la convergencia estructural del Mercosur (FOCEM)-Mercosur, convenio FOCEM N03/11 Proyecto "Investigación, Educación y Biotecnologías Aplicadas a la Salud (COF 03/11).

Declaración de conflictos de intereses: Los autores declaran no tener conflicto de intereses.

\section{FINANCIACIÓN}

La presente investigación no ha recibido ayudas específicas provenientes de agencias del sector público, sector comercial o entidades sin ánimo de lucro.

\section{REFERENCIAS BIBLIOGRÁFICAS}

1. Daniels CC, Rogers PD, Shelton CM. A review of pneumococcal vaccines: current polysaccharide vaccine recommendations and future protein antigens. J Pediatr Pharmacol Ther. 2016; 21: 27-35. Doi:10.5863/1551-6776-21.1.27.

2. Nagaraj S, Kalal BS, Manoharan A, Shet A. Streptococcus pneumoniae serotype prevalence and antibiotic resistance among young children with invasive pneumococcal disease: experience from a tertiary care center in South India. Germs. 2017; 7(2): 78-85. . doi:10.18683/germs.2017.1112.

3. Geno KA, Gilbert GL, Song JY, Skovsted IC, Klugman KP, Jones $C$, et al. Pneumococcal Capsules and Their Types: Past, Present, and Future. Clin Microbiol Rev. 2015; 28(3): 871-99. Doi: 10.1128/CMR.0002415.

4. Morales $M$, Ludwig G, Ercibengoa M, Esteva $C$, Sánchez-Encinales $V$, Alonso $M$, et al. Changes in the serotype distribution of Streptococcus pneumoniae causing otitis media after PCV13 introduction in Spain. PLoS ONE. 2018; 13(12): e0209048. Doi: 10.1371/journal.pone.0209048.

5. Parra EL, De La Hoz F, Díaz PL, Sanabria $\mathrm{O}$, Realpe ME, Moreno J. Changes in Streptococcus pneumoniae serotype distribution in invasive disease and nasopharyngeal carriage after the heptavalent pneumococcal conjugate vaccine introduction in Bogotá, Colombia. Vaccine. 2013; 31(37): 4033-38. Doi: 10.1016/j.vaccine.2013.04.074.

6. Balsells E, Guillot L, Nair H, Kyaw MH. Serotype distribution of Streptococcus pneumoniae causing invasive disease in children in the post-PCV era: A systematic review and meta-analysis. PLoS One. 2017; 12(5):e0177113.

Doi:10.1371/journal.pone.0177113.

7. Karcic E, Aljicevic M, Bektas S, Karcic B. Antimicrobial susceptibility/resistance of Streptococcus pneumoniae. Mater
Sociomed. 2015; 27: 180-84. Doi:10.5455/msm.2015.27.180-184.

8. Intra J, Besana S, Savarino C, Brambilla P. Serotype Distribution and Antimicrobial Resistance of Streptococcus pneumoniae Invasive Isolates Collected at the Italian Hospital of Desio, Lombardy, from 2008 to 2016. Front Public Health. 2017; 5:169. Doi:10.3389/fpubh.2017.00169.

9. Dirección General de Vigilancia de la Salud. Meningoencefalitis. Disponible en http://vigisalud.gov.py/sistemas/menin/\# . 2018. Citado 28 Agosto 2019.

10. Chamorro $G$, León $M$, Kawabata $A$, Franco R, Gómez G, Quiñónez B et al. Prevalencia de serotipos y resistencia a antibióticos betalactámicos de Streptococcus pneumoniae en menores de 6 años. Paraguay 2000 a 2008. Rev. Par. Epidemiol. 2011; 2 (1): 11-17.

11. León ME, Kawabata A, Nagai M, Rojas L, Chamorro G, Gómez G, et al. Streptococcus pneumoniae causante de enfermedad neumocócica invasivas en adultos. Paraguay (2013-2018). Rev. Soc. Cient. Paraguay, 2018; 23 (2): 263-74. Doi: $10.32480 /$ rscp. 2018-23-2.263-274.

12. Gabastou JM, Agudelo CI, Brandileone MCC, Castañeda E, Lemos APS, Di Fabio $\mathrm{JL}$, et al. Caracterización de aislamientos invasivos de $S$. pneumoniae, $H$. influenzae y $N$. meningitidis en América Latina y el Caribe: SIREVA II, 2000-2005. Rev Panam Salud Publica. 2008; 24(1):1-15. Retrieved from

http://www.scielosp.org/scielo.php?script= sci_arttext\&pid=S102049892008000700001.

13. Organización Panamericana de la Salud. Procedimientos para el diagnóstico de Neumonías y Meningitis Bacterianas y la caracterización de cepas de Streptococcus pneumoniae y Haemophilus influenzae, SIREVA II. 2012. Disponible en: https://www.paho.org/hq/dmdocuments/2 
012/PAHO-Manual-Neumo-HaemophilusSIREVA-2012.pdf. Citado 10 Mayo 2019.

14. CLSI. M100Ed28E. Performance Standards for Antimicrobial Susceptibility Testing: 28th Informational Supplement. Wayne, PA: Clinical and Laboratory Standards Institute; 2018.

15. Organización Panamericana de la Salud. Informe Regional de SIREVA II, 2014. Datos por país y por grupos de edad sobre las características de los aislamientos de Streptococcus pneumoniae, Haemophilus influenzae y Neisseria meningitidis en procesos invasores. Documentos técnicos. Regulaciones Sanitarias Internacionales, Alerta y Respuesta y Enfermedades Epidémicas (HSD/IR). Washington, D.C. OPS; 2015. http://iris. paho.org/xmlui/handle/1234567 89/33875. Citado 4 Junio 2019.

16. Parra EL, Ramos V, Sanabria O, Moreno J. Serotype and genotype distribution among invasive Streptococcus pneumoniae isolates in Colombia, 2005-2010. PLoS One. 2014; 9(1): e84993. Published 2014 Jan 8. Doi:10.1371/journal.pone.0084993.

17. Regis Leite C, Azevedo J, Santos Galvão V, Moreno-Carvalho O, Neves Reis J, Nascimento-Carvalho C. Clinical and bacteriological characteristics of invasive pneumococcal disease after pneumococcal 10-valent conjugate vaccine implementation in Salvador, Brazil. Braz J Infect Dis. 2016; 20(1): 56-60. Doi: 10.1016/j.bjid.2015.10.005.

18. Ruvinsky RO, Regueira M, Fossati MS, Gagetti $P$, Pace J, Rodríguez $M$ et al. Surveillance of invasive in Streptococcus pneumoniae in Argentina 1994-2007: Changes in serotype distribution, serotype coverage of pneumococcal conjugate vaccines and antibiotic resistance. Journal of Pediatric Infectious Diseases. 2010; 5: 263-69. doi: 10.3233/JPI-2010-026.

19. Mott M, Caierao J, da Cunha G, Rodrigues Perez LR, Matusiak R, Ruschel Pilger de Oliveira KR et al. Susceptibility profiles and correlation with pneumococcal serotypes soon after implementation of the 10-valent pneumococcal conjugate vaccine in Brazil. International Journal of Infectious Diseases. 2014: 20: 47-51. Doi: 10.1016/j.ijid.2013.11.009.

20. Castañeda E, Agudelo C, De Antonio R, Rosselli D, Calderon C, Ortega E. Streptococcus pneumoniae serotype 19A in Latin America and the Caribbean: a systematic review and meta-analysis, 1990-2010. BMC Infect Dis. 2012; 12:124. Doi: $10.1186 / 1471-2334-12-124$

21. Agudelo CI, De Antonio R, Castañeda E. Streptococcus pneumoniae serotype 19A in Latin America and the Caribbean 20102015: A systematic review and a time series analysis. Vaccine. 2018; 36 (32):
$4861-74$.

Doi:

10.1016/j.vaccine.2018.06.068.

22. Brandileone MC, Almeida S, Minamisava R, Andrade AL. Distribution of invasive Streptococcus pneumoniae serotypes before and 5 years after the introduction of 10 -valent pneumococcal conjugate vaccine in Brazil. Vaccine. 2018; 36 (19): 2559-66. Doi: $10.1016 / j$.vaccine.2018.04.010.

23. Isaacman $D$, McIntosh ED, Reinert RR. Burden of invasive pneumococcal disease and serotype distribution among Streptococcus pneumoniae isolates in young children in Europe: impact of the 7valent pneumococcal vaccine and considerations for future conjugate vaccines. Int] Infect Dis 2010; 14:e197209. Doi: 10.1016/j.ijid.2009.05.010.

24. Burgos J, Lujan $M$, Larrosa $M N$, Fontanals $D$, Bermudo G, Planes AM, et al. Risk factors for respiratory failure in pneumococcal pneumonia: the importance of pneumococcal serotypes Eur Respir J. 2014; 43: 545-53. Doi: $10.1183 / 09031936.00050413$.

25. Linley E, Bell A, Gritzfeld JF and Ray Borrow R. Should Pneumococcal Serotype $3 \mathrm{Be}$ Included in Serotype-Specific Immunoassays?. Vaccines. 2019; 7(1): 4. Doi: 10.3390/vaccines7010004.

26. Caierão J, Hawkins $P$, Sant'anna $F H$, da Cunha GR, d'Azevedo PA, McGee $L$, et al. Serotypes and Genotypes of Invasive Streptococcus pneumoniae Before and After PCV10 Implementation in Southern Brazil. PLoS ONE. 2014; 9(10): e111129. Doi: $10.1371 /$ journal.pone.0111129.

27. Carnalla-Barajas $M N$, Soto-Noguerón $A$, Miguel Angel Sánchez-Alemán MA, Solórzano-Santos $F$, Velazquez-Meza ME, Echániz-Aviles $G$, and Grupo SIREVAMexico. Changing trends in serotypes of $\mathrm{S}$. pneumoniae isolates causing invasive and non-invasive diseases in unvaccinated population in Mexico (2000-2014). International Journal of Infectious Diseases. 2017; 58: 1-7. Doi: 10.1016/j.ijid.2017.02.005.

28. Hackel M, Lascols C, Bouchillon S, Hilton B, Morgenstern D, Purdy J. Serotype prevalence and antibiotic resistance in Streptococcus pneumoniae clinical isolates among global populations. Vaccine. 2013; 31(42): 4881-87. Doi: $10.1016 /$ j.vaccine. 2013.07

29. Medeiros MI, Almeida SC, Bokermann S, Watanabe E, Guerra ML, Andrade D. Antimicrobial susceptibility of Streptococcus pneumoniae isolated from patients in the northeastern macroregion of São Paulo state, Brazil, 1998-2013. J. Bras. Patol. Med. Lab. [Internet]. 2017 June [cited 2019 June 22]; 53 (3): 177182. Available from: http://www.scielo.br/scielo.php?script=sci arttext\&pid=S1676- 
24442017000300177\&lng=en. http://dx.doi.org/10.5935/16762444.20170029.

30. Castañeda E, Agudelo CI, Regueira M, Corso A, de Cunto Brandileone MC, Brandao AP, et al. Laboratory- based surveillance of Streptococcus pneumoniae invasive disease in children in 10 latin American countries: a SIREVA II project, 2000-2005. Pediatr Infect Dis J. 2009; 28(9): e265-e270. Doi:10.1097/inf.0b013e3181a74b22

31. Jones RN, Sader HS, Mendes RE, Flamm RK. Update on antimicrobial susceptibility trends among Streptococcus pneumoniae in the United States: Report of ceftaroline activity from the SENTRY Antimicrobial Surveillance Program (1998-2011). Diagn Microbiol Infect Dis. 2013; 75:107-9. Doi: 10.1016/j.diagmicrobio.2012.08.024.

32. Grenón SL, Salvi Grabulosa MC, Regueira MM, Fossati MS, von Specht $\mathrm{MH}$.
Pneumococcal meningitis in children under 15 years of age in Misiones (Argentina). Sixteen year's epidemiological surveillance. Rev Argent Microbiol. 2014; 46:14-23. Doi: 10.1016/S0325-7541(14)70042-2.

33. Imöhl $M$, Reinert $R R$, Tulkens $P M$, van der Linden $\quad M$. Penicillin susceptibility breakpoints for Streptococcus pneumoniae and their effect on susceptibility categorisation in Germany (1997-2013). Eur J Clin Microbiol Infect Dis. 2014; 33: 2035-40. Doi: 10.1007/s10096-014-2174$\mathrm{Z}$.

34. Jin $\mathrm{P}, \mathrm{Wu} \mathrm{L}$, Oftadeh $\mathrm{S}$, Kudinha $\mathrm{T}$, Kong $\mathrm{F}$, Zeng $Q$. Using a practical molecular capsular serotype prediction strategy to investigate Streptococcus pneumoniae serotype distribution and antimicrobial resistance in Chinese local hospitalized children. BMC Pediatr. 2016; 16:53. Doi: 10.1186/s12887-016-0589-7. 